(2) Open Access Full Text Article

ORIGINAL RESEARCH

\title{
Serum Lactate Level Predicts Short-Term and Long-Term Mortality of HBV-ACLF Patients: A Prospective Study
}

This article was published in the following Dove Press journal:

Therapeutics and Clinical Risk Management

\section{Yuan Nie (iD) \\ Yue Zhang* \\ Lin-xiang Liu \\ Xuan Zhu}

Department of Gastroenterology, The First Affiliated Hospital of Nanchang University, Nanchang, Jiangxi, People's Republic of China

*These authors contributed equally to this work
Correspondence: Xuan Zhu

Department of Gastroenterology, The First Affiliated Hospital of Nanchang University,

No. 17, Yongwaizhengjie Road, Donghu

District, Nanchang 330006, Jiangxi, People’s

Republic of China

Email jyyfyzx@163.com
Background: Acute chronic liver failure (ACLF) is a high-mortality disease characterized by rapid deterioration of liver function and multiple organ failure. The aim of this study was to assess the short-term and long-term predictive values of serum lactate in HBV-ACLF patients to facilitate early treatment and thereby improve patient survival.

Methods: We conducted a single-center, observational prospective study of 108 hospitalized patients. Biochemical examination and demographic data were obtained within 24 hours of admission. Logistics analysis was used to determine whether serum levels were independently for prognosis of HBV-ACLF patients. The area under ROC curve evaluates the prediction accuracy compared to the existing score.

Results: Serum lactate levels in nonsurviving patients were significantly higher than those in surviving patients. Logistics analysis demonstrated that serum lactate was an independent risk factor for 28-day, 3-month, and 6-month mortality. ROC curve evaluates the prediction efficiencies of serum lactate for 28-day, 3-month, and 6-month mortality. The AUROCs of new scores by adding lactate (Child-Pugh+ lactate score, MELD+ lactate score, MELD-Na+ lactate score, CLIF-C OF+ lactate score, CLIF-SOFA+ lactate score, CLIF-C ACLF+ lactate score) were superior to those of existing scores, particularly the MELD score and MELD-Na score $(\mathrm{P}<0.05)$ at all time points.

Conclusion: Serum lactate can be used as an effective indicator to predict the short-term and long-term mortality in HBV-ACLF patients, and the predictive value of the MELD score and MELD-Na was improved by adjusting for lactate. Lactate testing at admission can be beneficial in prognostic assessment and clinical decision-making.

Keywords: acute-on-chronic liver failure, HBV, prognosis, lactate, scoring model

\section{Introduction}

Acute-on-chronic liver failure (ACLF) is a high-mortality disease characterized by acute liver function deterioration, hepatic or extrahepatic organ failure based on chronic liver disease. The mortality rate after integrated medical treatment can reach $50-90 \%{ }^{1}$ In European and American populations, the leading etiology of ACLF is alcoholic cirrhosis, followed by hepatitis $\mathrm{C}$ virus (HCV) infection, and the leading inducement of ACLF is bacterial infection and sepsis, followed by alcohol consumption. $^{2}$ In the Asia-Pacific region, the main cause of ACLF is hepatitis $\mathrm{B}$ virus (HBV) infection. Enhanced HBV replication caused by spontaneous activation, virus mutation and drug resistance are the main driver of $\mathrm{ACLF}^{3}$ Although liver transplantation can effectively improve liver failure, it is not widely used in 
clinical practice because of limited liver resources and high costs. Patients with advanced liver failure often miss the opportunity for liver transplantation due to poor surgical tolerance. Accurate evaluation and timely intervention are crucial to improve the prognosis of patients with ACLF. At present, most of the prognostic scoring models used in clinical practice, including the ChildTurcotte-Pugh (CTP) score, the model for end-stage liver disease (MELD) score, the MELD combined with serum sodium concentration (MELD-Na) score, the chronic liver failure consortium organ function (CLIF-C OF), the CLIF$\mathrm{C}$ sequential organ failure assessment (CLIF-SOFA) score, the CLIF-C ACLF, and the North American Consortium for the Study of End-Stage Liver Disease (NACSELD)-ACLF score were established by European and American countries. However, they have some obvious deficiencies and restrictions. Therefore, a simple and practicable parameter is necessary to predict prognosis and guide the choice of therapeutic measures.

Lactate is an intermediate product of anaerobic metabolism and is a marker of tissue hypoxia or metabolic changes due to stress reactions according to the release of epinephrine. ${ }^{4}$ A large number of animal experiments and clinical studies have shown that the lactate level has good performance in assessing the development of critically ill patients and predicting the prognosis of patients. The risk of death increases with increasing lactate levels. Lactate is an independent risk factor for death in severe patients, and even the only. ${ }^{5}$ Odam SM et al found that the mortality rate was $5.4 \%$ when lactate was less than 2.5 $\mathrm{mmol} / \mathrm{L}, 6.4 \%$ when lactate was $2.5-4 \mathrm{mmol} / \mathrm{L}, 18.8 \%$ when lactate was more than $4.0 \mathrm{mmol} / \mathrm{L}{ }^{6}$ The change in lactate in patients with normal vital signs can also help determine the severity of the injury. ${ }^{7}$ Lactate levels and clearance were independently correlated with short-term mortality in critically ill patients with cirrhosis, and the predictive value of lactate-adjusted score was significantly better than the existing scoring system at predicting mortality8. However, the study population was mainly Caucasian in Western countries. The purpose of this study was to evaluate the prognostic value of lactate in HBV-ACLF patients in order to guide clinical practice.

\section{Patients and Methods}

\section{Study Population}

This was a single-center, observational prospective cohort study conducted at a large tertiary public hospital in China between November 2013 and October 2019. The study protocol was approved by institutional ethics committee, and all participants obtained informed written consent.

The inclusion criteria were as follows: $\geq 18$ years of age and a diagnosis of cirrhosis and organ failure (defined by the CLIF-C SOFA score). ${ }^{2}$ The exclusion criteria were as follows: infection with human immunodeficiency virus; hepatocellular carcinoma that did not meet the Milan criteria or advanced stage cancer; other tumors except for hepatocellular carcinoma; previous liver transplantation; complications with other severe chronic extrahepatic diseases, such as bacterial infections, bacterial infections, renal failure, heart failure; taking drugs that affect the results of lactate levels, such as metformin, alcohol, adrenaline, catecholamine. Please check it in section of study population.

\section{Study Protocols}

Patients with HBV-ACLF were enrolled in the study when they were hospitalized. Demographic, treatment history, physical examination, laboratory test and radiological examination data were obtained from electronic medical records. Clinical and laboratory information, including age, sex, etiology of cirrhosis, comorbidities, laboratory parameters, events of organ failure, blood test was collected. Lactate was measured from the first radial or femoral artery within 24 hours of admission. Blood samples were collected in heparinized blood gas syringes and measured by blood gas analyzer (ABL 800 Series; Radiometer, Denmark) using Enzyme electrode method at $37^{\circ} \mathrm{C}$. The patients were followed for 6 months to obtain survival information.

\section{Definitions}

Patients with chronic HBV infection were confirmed by the detection of Hepatitis B surface antigen ( $\mathrm{HBsAg}$ ) positivity for more than 6 months. According to liver failuresequential organ failure assessment, ACLF is defined as acute decompensated patients with organ failure. ${ }^{2}$ Liver cirrhosis was diagnosed by the presence of ascites, hepatic encephalopathy (HE), variceal bleeding, radiologic imaging, hepatorenal syndrome, or liver biopsy, if available. ${ }^{9}$ The degree of ascites was diagnosed using the criteria proposed by the International Ascites Club and American Association for the Study of Liver Disease. ${ }^{10}$ The CTP score was calculated according to total bilirubin (TBil), albumin (ALB), international normalized ratio (INR), HE, ascites. ${ }^{11}$ The formula of MELD score: $3.78 \times \mathrm{Ln}(\mathrm{TBil} \mu \mathrm{mol} / \mathrm{L})+11.2 \times \mathrm{Ln}$ 
$(\mathrm{INR})+9.57 \times \mathrm{Ln}($ creatinine $\mu \mathrm{mol} / \mathrm{L})+6.43 \times($ constant for liver disease etiology $=0$ if cholestatic or alcoholic, otherwise $=1){ }^{12}$ The MELD-Na score is calculated as follows: MELD-Na-[0.025 $\times$ MELD $\times(140-N a)]+140 .{ }^{13}$ The CLIF-C OF score is the simplified calculations based on the CLIFSOFA score. ${ }^{14}$ The CLIF-C ACLF score was calculated as follows: $10 \times[0.33 \times \mathrm{CLIF}-\mathrm{C} \quad \mathrm{OF}+0.04 \times$ age $+0.63 \times \log$ (white-cell count)-2]. ${ }^{14}$ The NACSELD-ACLF score was defined by two or more extrahepatic organ failures. ${ }^{15}$

\section{Statistical Analysis}

All statistical analyses were performed using SPSS software version 20.0 (SPSS Inc., Chicago, IL). Continuous variables and Categorical variables were compared with the MannWhitney $U$-test or chi-squared tests. Receiver operating curves (ROC) were used to measure the performance of the score in predicting 28-day, 3-month, and 6-month mortality in patients with HBV-ACLF. DeLong test was performed to compared the areas under ROC curve. All statistical testing was performed at a two-tailed $\alpha$ level of 0.05 .

\section{Results}

\section{Baseline Characteristics of the Included Patients}

In total, 108 patients with HBV-ACLF were admitted to the hospital between November 2013 and October 2019. During the 28-day study period, 4 patients were excluded for loss to follow-up within 28 days, 6 patients were excluded for missing test results, and 1 patient was excluded for death within 24 hours. There were 97 patients included in the analysis of the outcomes at 28 days. Three patients were excluded for loss to follow-up between 28 days and 3 months; therefore, 94 patients were included in the complete follow-ups at 3 months and 6 months. The flowchart is shown in Figure 1, and the demographic and biochemical characteristics of the study population are outlined in Table 1. The mean ( \pm standard deviation) age of the 108 patients was $57.1( \pm 11.9)$ years. The majority of the patients were male (720/108). The leading cause of decompensation events responsible for hospitalization was variceal bleeding (75/108), followed by HE (14/108), infection (11/102), ascites (6/102), and others (1/102). The 28-day and 3-month analyses found that the distributions of patients who were included in the complete follow-ups at 28 days and at 3 months were similar in terms of ascites grade, ACLF grade, and treatment strategy. 49, 61, and 62 patients died within 28 days, 3 months, and 6 months, respectively. The leading causes of death at 6 months were hemorrhagic shock (22/62), followed by HE (15/62), respiratory failure $(12 / 62)$, infectious shock (6/62), liver failure (4/62), and unknown (3/62). The causes of death at 28 days, 3 months, and 6 months are summarized in Supplementary Table 1.
108 patients with $\mathrm{ACLF}$ at admission between November 2013 and October 2019

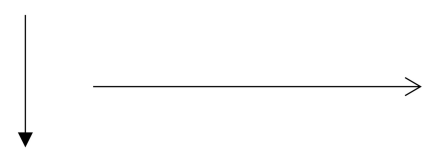

4 patients were exclude for loss of follow up within 28 days 6 patients were exclude for absence of test results 1 were exclude for death less than 24 hours
97 patients were included in the complete follow-up within 28 days

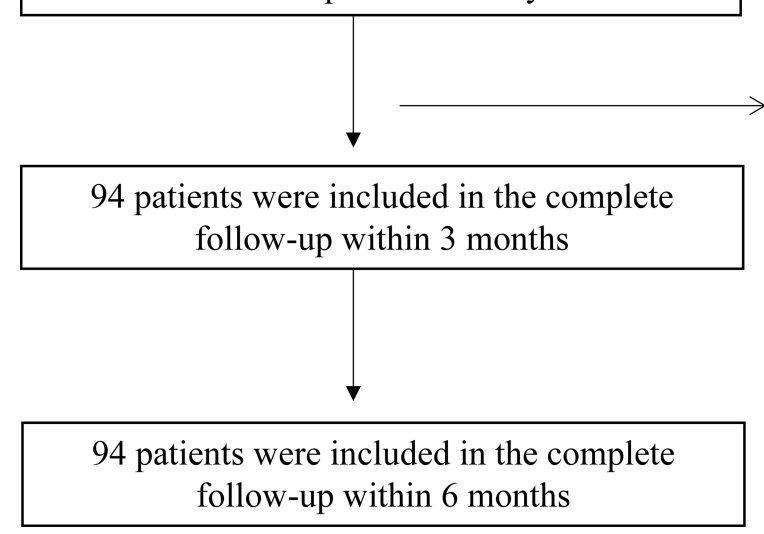

Figure I Patient selection flowchart.
3 patients were exclude for loss of follow up between 28 days and 3 months 
Table I Characteristics of Patients in the ACLF Cohort

\begin{tabular}{|c|c|c|c|}
\hline & $\begin{array}{l}\text { Patients with } \\
\text { ACLF } \\
\text { at Admission } \\
(n=108)\end{array}$ & $\begin{array}{l}\text { Patients in } \\
\text { Complete Follow- } \\
\text { Up } \\
\text { Within } 28 \text { Days } \\
(n=97)\end{array}$ & $\begin{array}{l}\text { Patients in Complete Follow-Up Within } 3 \\
\text { Months or } 6 \text { Months }(n=94)\end{array}$ \\
\hline Age, mean $\pm S D$ & $57.1 \pm 11.9$ & $58.1 \pm 11.7$ & $58.0 \pm 11.9$ \\
\hline Sex (male), n (\%) & $72(66.7 \%)$ & $64(66.0 \%)$ & 61 (64.9\%) \\
\hline $\begin{array}{l}\text { Hospitalization days, median } \\
\text { (IQR) }\end{array}$ & $4(I-10.5)$ & $4.5(1.25-11.0)$ & $5.0(1.0-11.0)$ \\
\hline \multicolumn{4}{|l|}{$\begin{array}{l}\text { Primary reason for } \\
\text { hospitalization, } \mathrm{n}(\%)\end{array}$} \\
\hline Variceal bleeding & 75 (69.4\%) & $70(72.2 \%)$ & 67 (71.3\%) \\
\hline Ascites & $6(5.6 \%)$ & $5(5.2 \%)$ & $5(5.3 \%)$ \\
\hline Hepatic encephalopathy & 14 (I3.0\%) & $13(13.4 \%)$ & $13(13.8 \%)$ \\
\hline Infection & II (10.2\%) & $8(8.2 \%)$ & $8(8.5 \%)$ \\
\hline Others & $\mathrm{I}(0.9 \%)$ & I (I.0\%) & $\mathrm{I}(\mathrm{I} .1 \%)$ \\
\hline \multicolumn{4}{|l|}{ ACLF grade, $n$ (\%) } \\
\hline ACLF grade I & 34 (3I.5\%) & 31 (32.0\%) & 30 (31.9\%) \\
\hline ACLF grade 2 & 47 (43.5\%) & $4 \mathrm{I}(42.2 \%)$ & 39 (4I.5\%) \\
\hline ACLF grade 3 & $27(25.0 \%)$ & $25(25.8 \%)$ & $25(26.6 \%)$ \\
\hline Endoscopic hemostasis, n (\%) & $5 \mathrm{I}(47.2 \%)$ & 48 (49.5\%) & 46 (48.9\%) \\
\hline \multicolumn{4}{|l|}{ The degree of ascites, $\mathrm{n}(\%)$} \\
\hline Mild & 15 (13.9\%) & $13(13.4 \%)$ & $12(12.8 \%)$ \\
\hline Moderate & 30 (27.8\%) & $29(29.9 \%)$ & 27 (28.7\%) \\
\hline Severe & $25(25.7 \%)$ & $24(24.5 \%)$ & $24(25.5 \%)$ \\
\hline Hepatocellular carcinoma, n (\%) & II (I0.2\%) & $10(10.3 \%)$ & $9(9.6 \%)$ \\
\hline Mechanical ventilation, n (\%) & $43(39.8 \%)$ & 39 (40.2\%) & $39(41.5 \%)$ \\
\hline Vasopressor use, n (\%) & 69 (63.9\%) & $64(66.0 \%)$ & 61 (64.9\%) \\
\hline
\end{tabular}

Abbreviations: ACLF, acute-on-chronic liver failure; SD, standard deviation; IQR, interquartile range.

\section{Comparing the Laboratory}

\section{Characteristics and Prognostic Model} Between Nonsurviving Groups and

\section{Surviving Groups}

The clinical and laboratory characteristics of the patients are listed in Supplementary Table 2. HBV-ACLF patients were divided into nonsurviving $(\mathrm{n}=49)$ and surviving groups ( $\mathrm{n}=48)$ according to 28 -day outcomes. The majority of nonsurvivors had higher grades than survivors, as reflected by bilirubin, INR, lactate, white blood cell count (WBC), alanine aminotransferase (ALT), aspartate aminotransferase (AST), prothrombin time (PT), CTP score, MELD score, CLIF-C OF score, CLIF-SOFA score,
CLIF-C ACLF score, and NACSELD-ACLF score $(\mathrm{P}<0.05)$; however, albumin levels were lower in nonsurvivors than in survivors $(\mathrm{P}<0.05)$. No significant differences in creatinine, platelets, mean arterial pressure (MAP), $\mathrm{PO}_{2} / \mathrm{FiO}_{2}$, alkaline phosphatase (ALP), gammaglutamyl transpeptidase (GGT), blood urea nitrogen (BUN), serum $\mathrm{Na}$, or MELD-Na score were observed $(\mathrm{P}<0.05)$. HBV-ACLF patients were divided into nonsurviving ( $\mathrm{n}=61)$ and surviving groups $(\mathrm{n}=33)$ according to 3 -month outcomes. There were statistically significant differences in bilirubin, lactate, WBC, albumin, BUN, the CTP score, the MELD score, the MELD-Na score, the CLIF-C OF score, the CLIF-SOFA score, the CLIF-C ACLF score, and the NACSELD-ACLF score between 
the groups $(\mathrm{P}<0.05)$. HBV-ACLF patients were divided into nonsurviving $(n=62)$ and surviving groups $(n=32)$ according to 6-month outcomes. The differences in bilirubin, lactate, WBC, albumin, blood urea nitrogen (BUN), the CTP score, the MELD score, the MELD-Na score, the CLIF-C OF score, the CLIF-SOFA score, the CLIF-C ACLF score, and the NACSELD-ACLF score $(\mathrm{P}<0.05)$ were statistically significant.

\section{Univariate and Multivariate Analyses for Mortality in HBV-ACLF Patients}

The univariate Cox proportional regression analysis of 28day mortality showed that the INR, lactate, albumin, ALT, ALP, serum Na, and PT were related risk factors $(\mathrm{P}<0.05)$ (Supplementary Table 3). Parameters whose P values were less than 0.1 in the univariate Cox regression were included in the multivariate Cox regression analysis, which showed that lactate, ALP, and serum Na were independent risk factors for 28-day mortality (HR: 1.196, 95\% CI:
1.052-1.377; HR: 1.009, 95\% CI: 1.001-1.012; HR: 1.041 95\% CI: 1.003-1.161, respectively). The univariate Cox regression analysis of 3-month mortality showed that lactate and albumin were related risk factors $(\mathrm{P}<0.05)$ and confirmed that lactate was an independent risk factor (HR 1.166, 95\% CI: 1.013-1.289) (Table 2). The univariate Cox regression analysis of 6-month mortality showed that bilirubin, lactate, and albumin were related risk factors $(\mathrm{P}<0.05)$ and confirmed that lactate was an independent risk factor (HR: 1.181, 95\% CI: 1.022-1.371) (Supplementary Table 3).

\section{Predictive Value for Mortality in HBV-ACLF Patients}

ROC curve analysis was performed to evaluate the accuracy of the CTP score, MELD score, MELD-Na score, CLIF-C OF score, CLIF-SOFA score, CLIF-C ACLF score, and lactate in predicting 28-day, 3-month, and 6-month mortality (Table 2). Lactate at admission was predictive of mortality at 28 days, 3 months, and 6 months in DeCi patients (AUROC:

Table 2 The Efficacy of the Various Systems for Prediction 28-Day, 3-Month and 6-Month Mortality

\begin{tabular}{|c|c|c|c|c|c|}
\hline Prognostic Score & ROC Area & P-value & Cut-Off Point & Sensitivity (\%) & Specificity (\%) \\
\hline \multicolumn{6}{|l|}{ 28-day mortality } \\
\hline CTP score & 0.710 & $<0.001$ & 10.00 & 57.91 & 74.42 \\
\hline MELD score & 0.675 & $<0.001$ & 22.00 & 84.42 & 59.61 \\
\hline MELD-Na score & 0.606 & $<0.001$ & 22.00 & 71.21 & 60.57 \\
\hline CLIF-C OF score & 0.775 & $<0.001$ & 10.00 & 84.67 & 57.45 \\
\hline CLIF-SOFA score & 0.809 & $<0.001$ & 10.00 & 77.82 & 74.72 \\
\hline CLIF-C ACLF score & 0.744 & $<0.001$ & 48.20 & 66.67 & 77.52 \\
\hline Lactate & 0.732 & $<0.001$ & 2.90 & 61.21 & 76.30 \\
\hline \multicolumn{6}{|l|}{ 3-month mortality } \\
\hline CTP score & 0.634 & $<0.001$ & 12.00 & 90.21 & 36.72 \\
\hline MELD score & 0.721 & $<0.001$ & 19.00 & 80.36 & 62.12 \\
\hline MELD-Na score & 0.665 & $<0.001$ & 20.50 & 74.19 & 62.13 \\
\hline CLIF-C OF score & 0.712 & $<0.001$ & 8.90 & 64.52 & 75.67 \\
\hline CLIF-SOFA score & 0.749 & $<0.001$ & 9.50 & 80.65 & 67.34 \\
\hline CLIF-C ACLF score & 0.729 & $<0.001$ & 48.20 & 74.19 & 75.02 \\
\hline Lactate & 0.702 & $<0.001$ & 2.80 & 64.54 & 75.10 \\
\hline \multicolumn{6}{|l|}{ 6-month mortality } \\
\hline CTP score & 0.647 & $<0.001$ & 12.00 & 92.86 & 36.14 \\
\hline MELD score & 0.712 & $<0.001$ & 19.00 & 82.14 & 60.49 \\
\hline MELD-Na score & 0.659 & $<0.001$ & 20.52 & 75.00 & 60.67 \\
\hline CLIF-C OF score & 0.721 & $<0.001$ & 9.50 & 64.27 & 73.87 \\
\hline CLIF-SOFA score & 0.745 & $<0.001$ & 9.50 & 82.14 & 65.57 \\
\hline CLIF-C ACLF score & 0.731 & $<0.001$ & 48.20 & 74.88 & 72.23 \\
\hline Lactate & 0.693 & $<0.001$ & 2.80 & 67.86 & 73.93 \\
\hline
\end{tabular}

Note: Bolded figures indicate statistically significant comparison $(P<0.05)$.

Abbreviations: CTP, Child-Turcotte-Pugh; MELD, model for end-stage liver disease; MELD-Na, model for end-stage liver disease-sodium; CLIF-C OF, CLIF consortium organ function; CLIF-SOFA, chronic liver failure-sequential organ failure assessment; CLIF-C ACLF, CLIF consortium acute-on-chronic liver failure. 
0.732, 95\% CI: 0.661-0.819; AUROC: 0.702, 95\% CI: 0.641-0.795; AUROC: 0.693, 95\% CI: 0.631-0.786, respectively). The cutoff value of lactate at 28 days was 2.9 , with a sensitivity of $60.00 \%$ and a specificity of $76.6 \%$. The cutoff value of lactate at 3 months was 2.9 , with a sensitivity of $64.52 \%$ and a specificity of $74.14 \%$, and the cutoff value of lactate at 6 months was 2.8 , with a sensitivity of $67.86 \%$ and a specificity of $73.77 \%$. The CTP score, MELD score, MELD-Na score, CLIF-C OF score, CLIF-SOFA score, and CLIF-ACLF score also had predictive value for mortality at 28 days, 3 months, and 6 months in HBV-ACLF patients (all $\mathrm{P}<0.001$ ).

\section{Predictive Value of Prognosis Models is Improved by Adjusting for Lactate}

To improve the predictive value, new scores (CTP+ lactate score, MELD+ lactate score, MELD-Na+ lactate score, CLIF-C OF+ lactate score, CLIF-SOFA+ lactate score, CLIF-C ACLF+ lactate score) were calculated by adding lactate to the MELD score and Child-Pugh score. The analysis of the AUROC at 28 days, 3 months, and 6 months showed that all the new scores had predictive value for mortality in HBV-ACLF patients (all $\mathrm{P}<0.001$ ) (Table 3). Comparison of the predictive value between the prognostic scores and the new scores including lactate showed that the AUROCs of all the new scores were superior to those of the prognostic scores at 28 days, 3 months, and 6 months. The AUROCs of the MELD+ lactate score and MELD-Na + lactate score were significantly superior to those of the MELD and MELD-Na scores at 28 days (difference between areas $=0.071,95 \%$ $\mathrm{CI}=0.013-0.128, \quad \mathrm{P}=0.014 ; \quad$ difference between areas $=0.062,95 \% \mathrm{CI}=0.012-0.112, \mathrm{P}=0.016$, respectively). The AUROCs of the MELD + lactate score and MELD-Na + lactate score were significantly superior than MELD score and MELD-Na score at 3 months (difference between areas $=0.051,95 \% \mathrm{CI}=0.001-0.103, \mathrm{P}=0.049$; difference between areas $=0.050,95 \% \mathrm{CI}=0.002-0.010$, $\mathrm{P}=0.040$, respectively). The AUROCs of the MELD + lactate score and MELD-Na+ lactate score were significantly superior than MELD score and MELD-Na score at 6 months (difference between areas $=0.052,95 \% \mathrm{CI}=0$.$001-0.105, \mathrm{P}=0.049$; difference between areas $=0.052$, $95 \% \mathrm{CI}=0.002-0.102, \mathrm{P}=0.043$, respectively). The ROC

Table 3 The Predictive Value of Six Score with Adding Lactate

\begin{tabular}{|c|c|c|c|c|c|}
\hline Prognostic Score & ROC Area & P-value & Cut-Off Point & Sensitivity (\%) & Specificity (\%) \\
\hline \multicolumn{6}{|l|}{ 28-day mortality } \\
\hline CTP+ Lactate & 0.762 & $<0.001$ & 15.02 & 75.62 & 72.42 \\
\hline MELD+ Lactate & 0.751 & $<0.001$ & 25.19 & 75.12 & 70.28 \\
\hline MELD-Na + Lactate & 0.671 & $<0.001$ & 24 & 58.08 & 77 \\
\hline CLIF-C OF+ Lactate & 0.771 & $<0.001$ & 17.1 & 91.41 & 53.59 \\
\hline CLIF-SOFA+ Lactate & 0.817 & $<0.001$ & 14.3 & 75.82 & 79.12 \\
\hline CLIF-C ACLF+ Lactate & 0.787 & $<0.001$ & 54.4 & 75.86 & 74.87 \\
\hline \multicolumn{6}{|l|}{ 3-month mortality } \\
\hline CTP+ Lactate & 0.710 & $<0.001$ & 15 & 77.81 & 64.09 \\
\hline MELD+ Lactate & 0.773 & $<0.001$ & 21 & 71.27 & 74.26 \\
\hline MELD-Na + Lactate & 0.719 & $<0.001$ & 24 & 64.83 & 72.81 \\
\hline CLIF-C OF+ Lactate & 0.726 & $<0.001$ & 17.2 & 90.62 & 45.23 \\
\hline CLIF-SOFA+ Lactate & 0.772 & $<0.001$ & 13.2 & 71.27 & 79.32 \\
\hline CLIF-ACLF+ Lactate & 0.761 & $<0.001$ & 51.7 & 64.42 & 79.71 \\
\hline \multicolumn{6}{|l|}{ 6-month mortality } \\
\hline CTP+ Lactate & 0.699 & $<0.001$ & 15 & 78.87 & 62.7 \\
\hline MELD+ Lactate & 0.762 & $<0.001$ & 20.1 & 71.56 & 74.72 \\
\hline MELD-Na + Lactate & 0.711 & $<0.001$ & 24 & 64.62 & 70.75 \\
\hline CLIF-C OF+ Lactate & 0.721 & $<0.001$ & 12 & 57.44 & 79.09 \\
\hline CLIF-SOFA+ Lactate & 0.759 & $<0.001$ & 13.2 & 71.73 & 77.61 \\
\hline CLIF-C ACLF+ Lactate & 0.757 & $<0.001$ & 51.8 & 64.82 & 77.45 \\
\hline
\end{tabular}

Note: Bolded figures indicate statistically significant comparison $(\mathrm{P}<0.05)$.

Abbreviations: CTP, Child-Turcotte-Pugh; MELD, model for end-stage liver disease; MELD-Na, model for end-stage liver disease-sodium; CLIF-C OF, CLIF consortium organ function; CLIF-SOFA, chronic liver failure-sequential organ failure assessment; CLIF-C ACLF, CLIF consortium acute-on-chronic liver failure. 

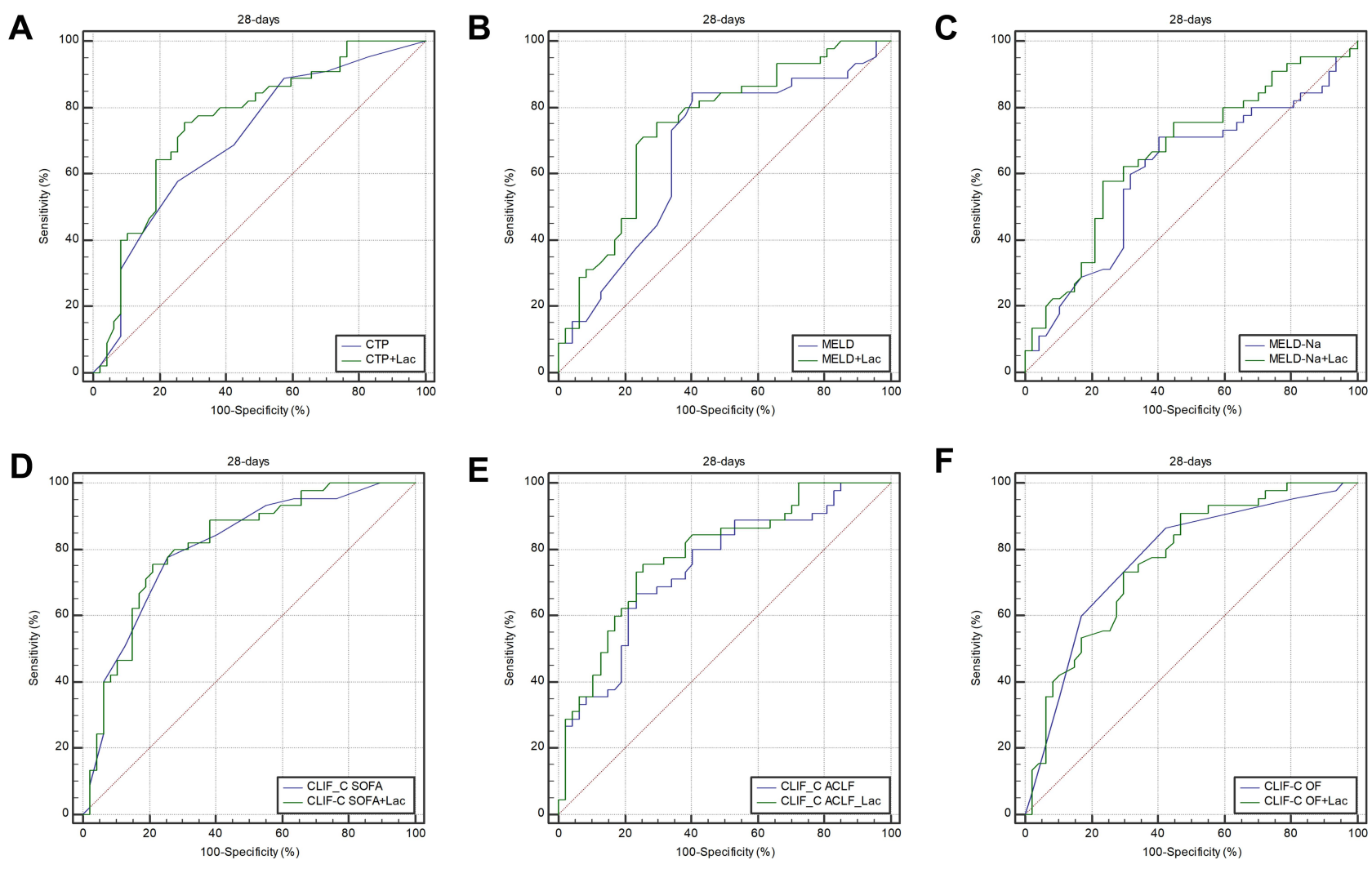

Figure $\mathbf{2}$ The ROC curves and comparisons of prognostic scores for 28 days.

curves and comparisons of prognostic scores are shown in Figure 2 (28 days), Figure 3 (3 months), Figure 4 (6 months) and Table 4.

\section{Discussion}

It is important to identify predictive factors that can distinguish patients with a high risk of death and to provide effective treatment as early as possible to reduce mortality, especially in those with high-mortality diseases. ACLF is high short-term mortality disease that can lead to serious life-threatening clinical complications, such as HE, hepatorenal syndrome, and upper gastrointestinal bleeding. Research on the pathogenesis, treatment and prognosis of ACLF is greatly affected by great differences in etiology and development between Asian countries and Western countries. The prognostic performance of specific patients is often unsatisfactory because of varying situations, such as disease stage, etiology, and complications. Seeking out a simple and useful parameter to predict the prognosis of HBV-ACLF patients in China is indispensable.

It should be noted that the characteristics of HBV-ACLF patients in the present study, particularly the etiology of ACLF patients, were different from those in Western countries. Most data from China shows that HBV is the primary etiological cause of cirrhosis in patients with ACLF. ${ }^{16}$ It is not surprising that the mortality of ACLF patients was high and was consistent with previous research. ${ }^{17-19}$ The short-term (28-day) mortality rate was $46.1 \%$, and the long-term (6-month) mortality rate was $59.8 \%$. The high-mortality rate appalls and saddens us and urges us to do meaningful things. Effective and inexpensive treatment strategies for patients with poor economic environment in developing countries are limited because of high liver transplant and hemodialysis costs. The economic burden caused by ACLF is still heavy. Predicting the prognosis of patients with ACLF may be more important than treatment from the perspective of health economics and low-income families.

Previous studies have revealed that the effectiveness of the CTP score, MELD score, MELD-Na score, CLIF-C OF score, CLIF-SOFA score, CLIF-C ACLF score and NACSELDACLF score in prognosis prediction in ACLF patients. ${ }^{11-}$ ${ }^{15,20-22}$ As expected, all the scores in the nonsurvival group were obviously higher than those in the nonsurvival group and had predictive value for mortality at 28 days, 3 months, and 6 months. This result suggests that all six scores can be applied to Asian populations. The CTP score and MELD score are used in liver function assessment, and is an independent related 


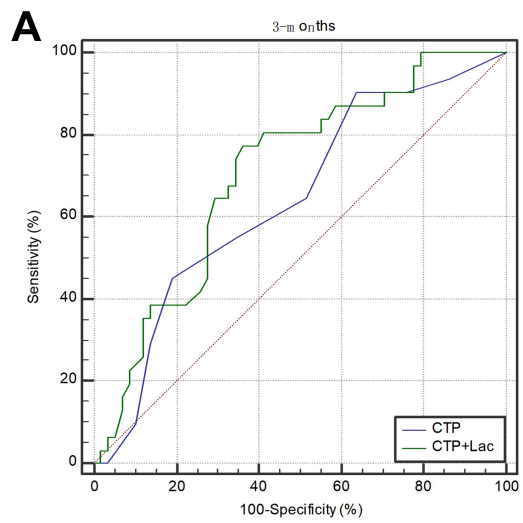

B

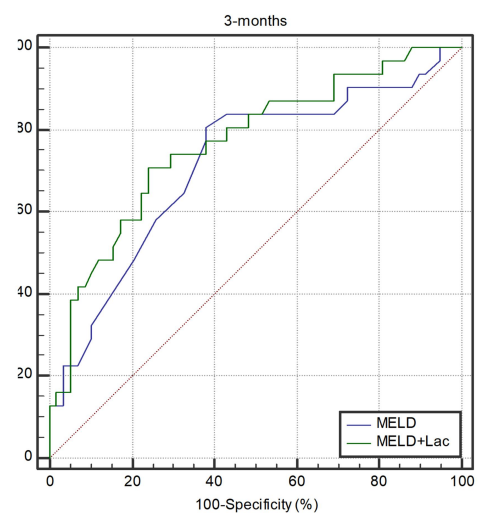

D

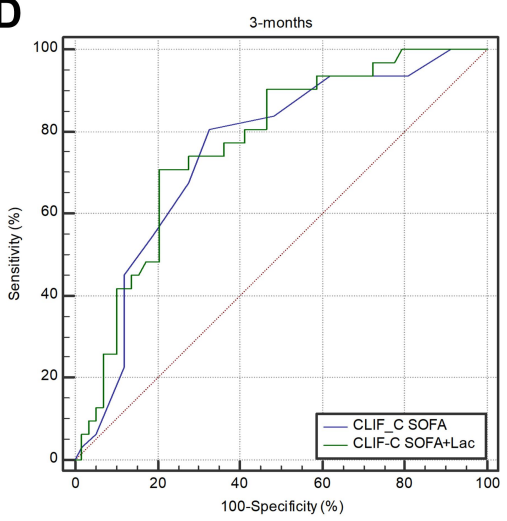

E

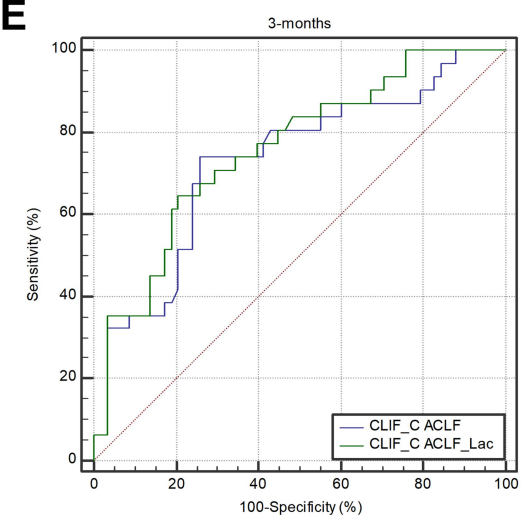

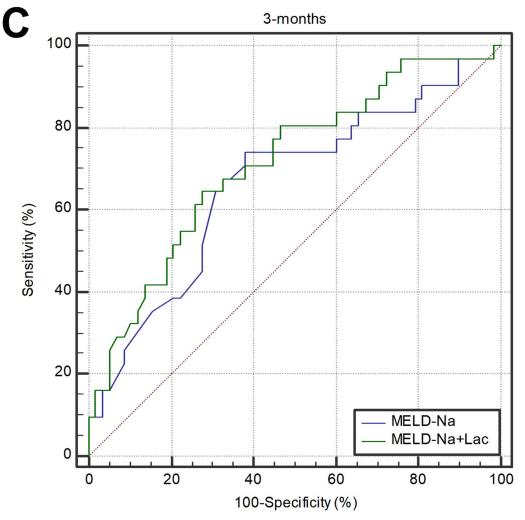

$\mathbf{F}$

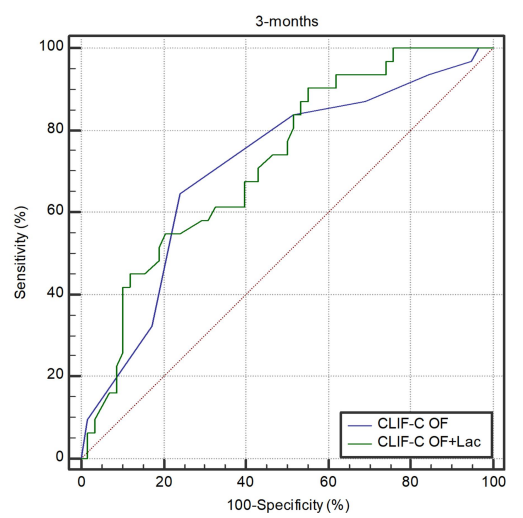

Figure 3 The ROC curves and comparisons of prognostic scores for 3 months.

factor for the prognosis of ACLF patients. Recently, the CLIFC OF score, CLIF-SOFA score, CLIF-C ACLF score, and NACSELD-ACLF scores were used to assess prognosis in ACLF patients. To our knowledge, although these scores have value for predicting prognosis of ACLF patients, different conclusions have been made regarding the scores with the best predictive values because different studies analyzed different research populations and observation times.

Multiple studies provide considerable evidence for the prognostic value of lactate in critically ill patients. ${ }^{23}$ Some studies have shown that lactate levels are associated with mortality in ICU DeCi patients. Andreas Drolz's study revealed that lactate appropriately reflected organ failure was independently associated with short-term mortality in DeCi patients after nearly one year of follow-up, and the performance of the CLIF-C ACLF score was significantly improved by adjusting for lactate. ${ }^{8}$ In Zhou XD's study of 949 critically ill cirrhotic patients with acute respiratory failure, a new score (ARF-CLIF-SOFA) considered serum lactate and had better accuracy for predicting 30-day, 90-day and 1-year mortality than the MELD score and CLIF-SOFA score. ${ }^{24}$ However, their study did not clearly reveal that lactate levels were associated with prognosis in $\mathrm{DeCi}$ patients in China. Considering the natural differences in etiologies, development and mechanisms of ACLF between Asian countries and Western countries, to confirm the prognostic value of lactate levels in Asian ACLF patients, we established a cohort of ACLF patients based on cirrhosis. First, we found that lactate was significantly higher in nonsurviving patients than in surviving patients (Supplementary Table 2), and univariate and multivariate analyses of mortality in DeCi patients were performed to confirm that lactate levels were an independent risk factor for death in patients with ACLF (Supplementary Table 3). Further, the predictive value of lactate levels at admission was determined (Table 2). The results showed that lactate levels at admission had good prediction accuracy for mortality in ACLF patients at 28 days, 3 months, and 6 months. The new score (Child-Pugh+ lactate score, MELD+ lactate score, MELD-Na+ lactate score, CLIF-C OF+ lactate score, CLIF-SOFA+ lactate score, CLIF$\mathrm{CACLF}+$ lactate score) also had improved prediction accuracy for mortality in ACLF patients at 28 days, 3 months, and 6 months (Table 3). Finally, a comparison of the prediction accuracy between the new score and score based on the same data was conducted. Our study indicated that the AUROC of 

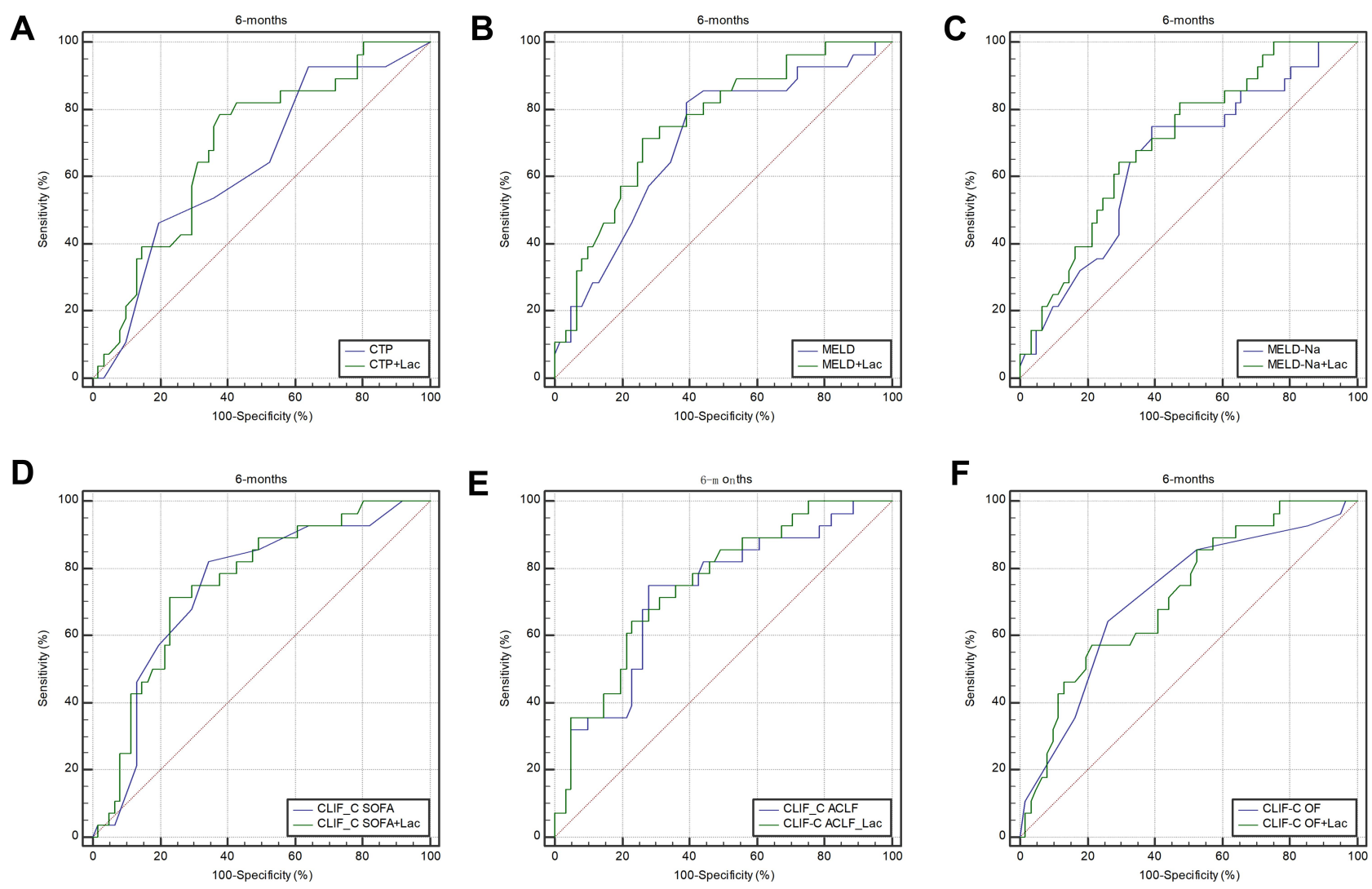

Figure 4 The ROC curves and comparisons of prognostic scores for 6 months.

the new score was higher than that of the standard score (Table 4 ), and the predictive values of the MELD score and MELD$\mathrm{Na}$ score were significantly improved by adding lactate at all time points. Our findings demonstrate that lactate is also a good and independent predictor of long-term outcomes in Chinese HBV-ACLF patients whose cirrhosis is mainly caused by viral hepatitis.

The underlying mechanisms by which serum lactate can predict the prognosis of patients with ACLF are not well defined. Lactate is an intermediate product of anaerobic metabolism. Some glucose in body tissues and organs, such as skeletal muscle, red blood cells, and brain tissue in the resting state, can ferment to form lactate during metabolism. ${ }^{25}$ Lactate is cleared at a near constant rate by three main pathways: gluconeogenesis, tricarboxylic acid circulation in the liver, and secretion by the kidney. Once there is an abnormality in either lactate production or elimination in the body, the rate of lactate production exceeds the elimination rate, leading to the accumulation of lactate in the body. ${ }^{26}$ The liver is responsible for up to $70 \%$ of whole-body lactate clearance. Hepatic impairment is associated with increased lactate levels due to impaired mitochondrial oxidation. ${ }^{27}$
Another reason for hyperlactatemia is an imbalance in microcirculatory blood oxygen. Cirrhosis often leads to the failure of visceral microvascular function, which reduces the supply of oxygen or nutrients to peripheral tissues. Elevated lactate levels are a sign of tissue hypoperfusion. $^{28}$ Clinical observation showed that the organ failure score was increased with increasing lactate levels. $^{29}$ Jansen TC et al confirmed that serum lactate is closely related to the multiple organ failure score. ${ }^{30}$ The MELD score and MELD-Na score are prognostic models for patients with cirrhosis and do not take into account inflammatory activation and organ failure associated with ACLF. This may be the main reason the predictive value of the new score (MELD+ lactate score, MELD-Na+ lactate score) is higher than that of the MELD score. Hence, we assume that the relationship between blood lactate and prognosis in DeCi patients is the result of comprehensive effects on metabolism and microcirculation.

This study also has some limitations. First, this study was a single-center, observational prospective cohort study in China. Some patients were lost follow-up leading to bias in participant selection. Second, the predictive role of dynamic changes of lactate is missing, as the long-term 
Table 4 Comparison of Prognostic Score with Prognostic Score by Adding Lactate

\begin{tabular}{|l|l|l|l|}
\hline Prognostic Score & Difference Between Areas & $\mathbf{9 5 \%} \mathbf{C l}$ & P-value \\
\hline 28-day mortality & & & \\
CTP + Lactate vs CTP & 0.05 I & $-0.028-0.130$ & 0.108 \\
MELD + Lactate vs MELD & 0.071 & $0.013-0.128$ & $\mathbf{0 . 0 1 4}$ \\
MELD-Na + Lactate vs MELD-Na & 0.062 & $0.012-0.112$ & $\mathbf{0 . 0 1 6}$ \\
CLIF-SOFA + Lactate vs CLIF-SOFA & 0.007 & $-0.055-0.070$ & 0.813 \\
CLIF-C ACLF + Lactate vs CLIF-C ACLF & 0.044 & $-0.001-0.090$ & 0.057 \\
CLIF-C OF + Lactate vs CLIF-C OF & 0.004 & $-0.066-0.074$ & 0.910 \\
\hline 3-month mortality & & & \\
CTP + Lactate vs CTP & 0.064 & $-0.015-0.143$ & 0.110 \\
MELD + Lactate vs MELD & 0.051 & $0.001-0.103$ & $\mathbf{0 . 0 4 9}$ \\
MELD-Na + Lactate vs MELD-Na & 0.050 & $0.002-0.010$ & $\mathbf{0 . 0 4 0}$ \\
CLIF-SOFA + Lactate vs CLIF-SOFA & 0.016 & $-0.048-0.080$ & 0.62 I \\
CLIF-C ACLF + Lactate vs CLIF-C ACLF & 0.029 & $-0.013-0.072$ & 0.180 \\
CLIF-C OF + Lactate vs CLIF-C OF & 0.013 & $-0.072-0.097$ & 0.767 \\
\hline 6-month mortality & & & \\
CTP + Lactate vs CTP & 0.054 & $-0.027-0.134$ & 0.193 \\
MELD + Lactate vs MELD & 0.052 & $0.001-0.105$ & $\mathbf{0 . 0 4 9}$ \\
MELD-Na + Lactate vs MELD-Na & 0.052 & $0.002-0.102$ & $\mathbf{0 . 0 4 3}$ \\
CLIF-SOFA + Lactate vs CLIF-SOFA & 0.011 & $-0.055-0.078$ & 0.743 \\
CLIF-C ACLF + Lactate vs CLIF-C ACLF & 0.025 & $-0.016-0.072$ & 0.205 \\
CLIF-C OF + Lactate vs CLIF-C OF & 0.002 & $-0.088-0.091$ & 0.970 \\
\hline Nof & & & \\
\hline
\end{tabular}

Note: Bolded figures indicate statistically significant comparison $(P<0.05)$.

Abbreviations: CTP, Child-Turcotte-Pugh; MELD, model for end-stage liver disease; MELD-Na, model for end-stage liver disease-sodium; CLIF-C OF, CLIF consortium organ function; CLIF-SOFA, chronic liver failure-sequential organ failure assessment; CLIF-C ACLF, CLIF consortium acute-on -chronic liver failure.

changes in serum lactate and lactate clearance were not routinely measured. Finally, we failed to assess the correlation between lactate and organ failure because of information about lack of organ failure was missing.

\section{Conclusion}

Many scores may be useful as predictors of mortality in hospitalized patients with HBV-ACLF, including the MELD score and Child-Pugh score. Our results indicate that lactate level independently predicts the short-term and long-term prognosis of HBV-ACLF patients. Serum lactate levels at admission had a predictive value for morbidity similar to those of the CTP score, MELD score, MELD-Na, CLIF-C OF score, CLIFSOFA score, and CLIF-C ACLF score in the Chinese population, and the predictive accuracy of the MELD score and MELD-Na were improved by including lactate.

\section{Abbreviations}

HBV, hepatitis B virus; LC, liver cirrhosis; HE, hepatic encephalopathy; MELD, model for end-stage liver disease; HBsAg, hepatitis B surface antigen; HRS, hepatorenal syndrome; ACLF, acute-on-chronic liver failure; ALT, alanine aminotransferase; AST, aspartate aminotransferase; ALB, albumin; TBil, total bilirubin; PT, prothrombin time; INR, international normalized ratio; WBC, white blood cell count; PLT, platelet; Cr, creatinine; ALP, alkaline phosphatase; GGT, gamma-glutamyl transpeptidase; AUROC, the areas under the receiver operating characteristic curve; CLIF-C OF, CLIF consortium organ function; CLIF-SOFA, chronic liver failuresequential organ failure assessment; CLIF-C ACLF, CLIF consortium acute-on-chronic liver failure.

\section{Data Sharing Statement}

The data sets generated and analyzed during this study are available from the corresponding author on reasonable request.

\section{Ethics Approval and Consent to Participate}

All procedures involving human participants were approved by the Ethics Committee of The First Affiliated Hospital of Nanchang University (No.2013-0103). The 
study was performed in accordance with Helsinki Declaration. All subjects signed informed consent and volunteered to participate in the study.

\section{Acknowledgments}

The authors are thankful to the anonymous reviewers for their comments.

\section{Funding}

This study was supported by the National Natural Science Foundation of China (grant no. 81960120) and the "GanPo Talent 555" Project of Jiangxi Province.

\section{Disclosure}

The authors declare that they have no competing interests. The funders had no role in the study design, data collection and analysis, decision to publish, or preparation of the manuscript.

\section{References}

1. Bernal W, Jalan R, Quaglia A, et al. Acute-on-chronic liver failure. Lancet. 2015;386(10003):1576-1587. doi:10.1016/S0140-6736(15) 00309-8.

2. Moreau R, Jalan R, Gines P, et al. Acute-on-chronic liver failure is a distinct syndrome that develops in patients with acute decompensation of cirrhosis. Gastroenterology. 2013;144(7):1426-1437. doi:10.1053/j.gastro.2013.02.042.

3. Wu T, Li J, Shao L, et al. Development of diagnostic criteria and a prognostic score for hepatitis B virus-related acute-on-chronic liver failure. Gut. 2018;67(12):2181-2191. doi:10.1136/gutjnl-2017314641.

4. Garcia-Alvarez M, Marik P, Bellomo R. Sepsis-associated hyperlactatemia. Crit Care. 2014;18(5):503. doi:10.1186/s13054-014 0503-3.

5. Vanni S, Viviani G, Baioni M, et al. Prognostic value of plasma lactate levels among patients with acute pulmonary embolism: the thrombo-embolism lactate outcome study. Ann Emerg Med. 2013;61 (3):330-338. doi:10.1016/j.annemergmed.2012.10.022.

6. Odom SR, Howell MD, Silva GS, et al. Lactate clearance as a predictor of mortality in trauma patients. $J$ Trauma Acute Care Surg. 2013;74(4):999-1004. doi:10.1097/TA.0b013e3182858a3e.

7. Paladino L, Sinert R, Wallace D, et al. The utility of base deficit and arterial lactate in differentiating major from minor injury in trauma patients with normal vital signs. Resuscitation. 2008;77(3):363-368. doi:10.1016/j.resuscitation.2008.01.022.

8. Drolz A, Horvatits T, Rutter K, et al. Lactate improves prediction of short-term mortality in critically ill patients with cirrhosis: a multinational study. Hepatology. 2019;69(1):258-269. doi:10.1002/ hep.30151.

9. Runyon BA, Practice Guidelines Committee AAftSoLD. Management of adult patients with ascites due to cirrhosis. Hepatology. 2004;39 (3):841-856. doi:10.1002/hep.20066.
10. Arroyo V, Gines P, Gerbes AL, et al. Definition and diagnostic criteria of refractory ascites and hepatorenal syndrome in cirrhosis. International ascites club. Hepatology. 1996;23(1):164-176. doi:10.1002/hep.510230122.

11. Pugh RN, Murray-Lyon IM, Dawson JL, et al. Transection of the oesophagus for bleeding oesophageal varices. Br J Surg. 1973;60 (8):646-649. doi:10.1002/bjs.1800600817.

12. Malinchoc M, Kamath PS, Gordon FD, et al. A model to predict poor survival in patients undergoing transjugular intrahepatic portosystemic shunts. Hepatology. 2000;31(4):864-871. doi:10.1053/he.2000.5852.

13. Biggins SW, Kim WR, Terrault NA, et al. Evidence-based incorporation of serum sodium concentration into MELD. Gastroenterology. 2006;130(6):1652-1660. doi:10.1053/j.gastro.2006.02.010.

14. Jalan R, Saliba F, Pavesi M, et al. Development and validation of a prognostic score to predict mortality in patients with acute-onchronic liver failure. $J$ Hepatol. 2014;61(5):1038-1047. doi:10.1016/j.jhep.2014.06.012.

15. Bajaj JS, O'Leary JG, Reddy KR, et al. Survival in infection-related acute-on-chronic liver failure is defined by extrahepatic organ failures. Hepatology. 2014;60(1):250-256. doi:10.1002/hep.27077.

16. Sarin SK, Kumar M, Eslam M, et al. Liver diseases in the asia-pacific region: a lancet gastroenterology \& hepatology commission. Lancet Gastroenterol Hepatol. 2020;5(2):167-228. doi:10.1016/S24681253(19)30342-5.

17. Sundaram V, Jalan R, Wu T, et al. Factors associated with survival of patients with severe acute-on-chronic liver failure before and after liver transplantation. Gastroenterology. 2019;156(5):1381-1391 e1383. doi:10.1053/j.gastro.2018.12.007.

18. Mahmud N, Kaplan DE, Taddei TH, et al. Incidence and mortality of acute-on-chronic liver failure using two definitions in patients with compensated cirrhosis. Hepatology. 2019;69(5):2150-2163. doi:10.1002/hep.30494.

19. Hernaez R, Kramer JR, Liu Y, et al. Prevalence and short-term mortality of acute-on-chronic liver failure: a national cohort study from the USA. $J$ Hepatol. 2019;70(4):639-647. doi:10.1016/j. jhep.2018.12.018.

20. Reverter E, Tandon P, Augustin S, et al. A MELD-based model to determine risk of mortality among patients with acute variceal bleeding. Gastroenterology. 2014;146(2):412-419 e413. doi:10.1053/j.gastro.2013.10.018.

21. Hernaez R, Sola E, Moreau R, et al. Acute-on-chronic liver failure: an update. Gut. 2017;66(3):541-553. doi:10.1136/gutjnl-2016-312670.

22. Dhiman RK, Agrawal S, Gupta T, et al. Chronic liver failure-sequential organ failure assessment is better than the asia-pacific association for the study of liver criteria for defining acute-on-chronic liver failure and predicting outcome. World J Gastroenterol. 2014;20(40):14934-14941. doi:10.3748/wjg.v20.i40.14934.

23. Jansen TC, van Bommel J, Schoonderbeek FJ, et al. Early lactate-guided therapy in intensive care unit patients: a multicenter, open-label, randomized controlled trial. Am J Respir Crit Care Med. 2010;182(6):752-761. doi:10.1164/rccm.200912-1918OC.

24. Zhou XD, Chen QF, Zhang MC, et al. Scoring model to predict outcome in critically ill cirrhotic patients with acute respiratory failure: comparison with MELD scoring models and CLIF-SOFA score. Expert Rev Gastroenterol Hepatol. 2017;11(9):857-864. doi:10.1080/ 17474124.2017.1338948.

25. Doherty JR, Cleveland JL. Targeting lactate metabolism for cancer therapeutics. J Clin Invest. 2013;123(9):3685-3692. doi:10.1172/ JCI69741.

26. Jeppesen JB, Mortensen C, Bendtsen F, et al. Lactate metabolism in chronic liver disease. Scand J Clin Lab Invest. 2013;73(4):293-299. doi:10.3109/00365513.2013.773591. 
27. Scheiner B, Lindner G, Reiberger $\mathrm{T}$, et al. Acid-base disorders in liver disease. $J$ Hepatol. 2017;67(5):1062-1073. doi:10.1016/j. jhep.2017.06.023.

28. Reddy AJ, Lam SW, Bauer SR, et al. Lactic acidosis: clinical implications and management strategies. Cleve Clin J Med. 2015;82 (9):615-624. doi:10.3949/ccjm.82a.14098.

29. McNelis J, Marini CP, Jurkiewicz A, et al. Prolonged lactate clearance is associated with increased mortality in the surgical intensive care unit. Am J Surg. 2001;182(5):481-485. doi:10.1016/s00029610(01)00755-3.
30. Jansen TC, van Bommel J, Woodward R, et al. Association between blood lactate levels, sequential organ failure assessment subscores, and 28-day mortality during early and late intensive care unit stay: a retrospective observational study. Crit Care Med. 2009;37(8):2369-2374. doi:10.1097/CCM.0b013e3181a0f 919.

\section{Publish your work in this journal}

Therapeutics and Clinical Risk Management is an international, peerreviewed journal of clinical therapeutics and risk management, focusing on concise rapid reporting of clinical studies in all therapeutic areas, outcomes, safety, and programs for the effective, safe, and sustained use of medicines. This journal is indexed on PubMed Central, CAS,
EMBase, Scopus and the Elsevier Bibliographic databases. The manuscript management system is completely online and includes a very quick and fair peer-review system, which is all easy to use. Visit http://www.dovepress.com/testimonials.php to read real quotes from published authors. 